\title{
Michael Schuenke, Erik Schulte, Udo Schumacher (Editors): Atlas of anatomy: head, neck, and neuroanatomy
}

\author{
Thieme publishers New York, 2016 . ISBN 978-1-62623-120-7
}

\author{
Sara Maria delle Acque Giorgio ${ }^{1} \cdot$ Luigi Mansi $^{1}$
}

Published online: 18 January 2017

(C) Springer-Verlag Berlin Heidelberg 2017

This second edition of volume 3 in the Thieme Atlas of Anatomy series covers anatomy of the neck, as well as anatomy of the head and neuroanatomy. The authors are Michael Schuenke, Erik Schulte, Udo Schumacher, working respectively at the Christina Albrecht University in Kiel, Johannes Gutenberg University in Mainz and University Medical Center in Hamburg-Eppendorf. The contribution as consulting editors of Brian R. MacPherson, from the University of Kentucky in Lexington, and of Cristian Stefan, from the Georgia Regents University in Augusta, is also recognized. The beautiful illustrations, rich of extraordinary detail, have been produced by Markus Voll and Karl Wesker.

Five hundred and fifty-five pages in large format include, with respect to the previous edition, also new exquisite and full-color anatomic illustrations, more than 1700 , for the head, neck, and neuroanatomy, each one accompanied by clarifying and concise text, as well as a substantial number of additional clinical correlations. Therefore, the book combines succinct explanatory text with the most beautiful, precise anatomical pictures.

Descriptions of anatomic structures and their relationships to one another, along with information on the development of the structures, anomalies, and common pathologies, appear in every chapter.

This format seems, at a first approach, perhaps too voluminous to be consulted quickly; nevertheless, it presents a selfcontained guide to a specific topic, strongly facilitating an easy didactic use. Furthermore, summary tables throughout the text appear ideal for rapid review.

Luigi Mansi

luigi.mansi@unina2.it

1 Università della Campania "Luigi Vanvitelli", Caserta, Italy
Access to head, neck, and neuroanatomy images on Winking Skull.com PLUS, featuring labels-on, labels-off functionality and timed self-tests are also available.

This atlas connects the basic science of anatomy to the clinical practice, and it is precious for students who are taking anatomy courses in addition to young medical doctors who need a more systemic and topographic anatomic education. In this sense it represents a useful publication also for physicians and residents involved in diagnostic imaging. Moreover cross-sections, X-rays, CT, and MRI scans vividly demonstrate clinical anatomy, and they make this atlas a helpful support for classroom and active dissection in the laboratory. Thus, we can define it as "a must-have book" for people who want to learn more and better by seeing structures rather than just reading about them.

This atlas is made up of 22 chapters divided into three sections: head and neck, neuroanatomy, and a glossary.

The first section starts with an overview of bones, ligaments, joints, and muscles, which make up the head and neck apparatus, and then it classifies and describes in detail the involved neurovascular structures.

The second section introduces the neurophysiology of the brain, and then it describes all the brain components: telencephalon, diencephalon, brainstem, cerebellum, and spinal cord, with dedicated chapters to meninges, the ventricular system, blood vessels. The third section is a useful glossary quickly allowing an overview of all the brain functional systems thanks to many clarifying diagrams. Finally, the atlas ends with references and a subject index.

This publication aims to become a standard guide for students in their initial approach to anatomy and a useful text to all medical doctors who want to improve their anatomic knowledge in the areas of the head and neck and in neuroanatomy. 